\title{
High-fidelity feedback-assisted parity measurement in circuit QED
}

\author{
L. Tornberg and G. Johansson \\ Department of Microtechnology and Nanoscience, MC2, Chalmers University of Technology, SE-41296 Gothenburg, Sweden
}

(Received 29 March 2010; revised manuscript received 20 May 2010; published 26 July 2010)

\begin{abstract}
We analyze a two-qubit parity measurement based on dispersive readout in circuit quantum electrodynamics. The back action on the qubits has two qualitatively different contributions. One is an unavoidable dephasing in one of the parity subspaces, arising during the transient time of switching on the measurement. The other part is a stochastic rotation of the phase in the same subspace, which persists during the whole measurement. The latter can be determined from the full measurement record using the method of state estimation. Our main result is that the outcome of this phase determination process is independent of the initial state in the state estimation procedure. The procedure can thus be used in a measurement situation where the initial state is unknown. We discuss how this feedback method can be used to achieve a high-fidelity parity measurement for moderate values of the cavity-qubit coupling strength. Finally, we discuss the robustness of the feedback procedure toward errors in the measurement record.
\end{abstract}

DOI: $10.1103 /$ PhysRevA.82.012329

PACS number(s): 03.67.Pp, 42.50.Dv, 02.30.Yy, 42.50.Pq

\section{INTRODUCTION}

In circuit QED (cQED) [1,2], superconducting qubits [3-5] are coupled to a microwave cavity, which allows for highfidelity qubit state control [6] and measurement [7-9], two ingredients required to achieve a scalable quantum information architecture [10]. In the dispersive regime, where the cavity and qubits are sufficiently detuned [1], the state of the qubits shifts the resonance frequency of the cavity, which can be detected by a homodyne measurement of the field state. This realizes a joint readout of the state of the qubits and allows for measurement of single as well as multiqubit operators [7-9]. Such a resource allows for measurement of the state parity, which in turn enables deterministic entanglement through measurement [11-13] and implementation of error correction protocols [14-16]. A true two-qubit parity measurement projects the state of the system on either the even-parity subspace spanned by $|g g\rangle$ and $|e e\rangle$ or the oddparity subspace spanned by $|g e\rangle$ and $|e g\rangle$, simultaneously allowing the observer to infer which of the two projections has occurred. The measurement is, however, not allowed to give information about the nature of the states within the respective subspaces as this would destroy the quantum superposition of the postmeasurement state [17]. In the dispersive readout, the entanglement between qubits and field allows the observer to infer the parity of the qubit state. This measurement has previously been analyzed by Lalumière et al. [13], where they find the same entanglement to give rise to an unavoidable back action causing dephasing within one of the parity subspaces. This severely limits the fidelity of the measurement and causes the postmeasurement state to be mixed and thus limited for further use in a computational context.

In this work, we extend the analysis of Lalumière et al. and show that the full measurement record from the homodyne current together with quantum feedback based on state estimation $[18,19]$ can be used to significantly reduce the unwanted dephasing caused by the measurement. This possibility was previously analyzed in a different setup where the homodyne detection could be regarded as a strong projective measurement [20]. For cQED, a weak measurement is more realistic, and in the present article, we extend the analysis to this situation using a quantum trajectories approach [21].

To exploit quantum feedback, it is necessary to use a nearly quantum-limited measurement system. The limiting element in CQED dispersive readout is the microwave amplifier. Amplifiers with noise temperatures of a few kelvins are commercially available. This noise temperature corresponds to adding 5-25 noise photons for each signal photon. However, the quantum limit has been reached using low-temperature superconducting amplifiers [22], and there is an ongoing effort to reach the quantum limit, also in the context of qubit measurements [23-25].

\section{DISPERSIVE READOUT AS A PARITY MEASUREMENT}

In this section, we lay out the essential idea behind the realization of a two-qubit parity measurement using dispersive readout in CQED [1,2]. For two qubits, the objective of a parity measurement is to distinguish between measurement results corresponding to states belonging to the two orthogonal subspaces $\mathcal{H}_{+}=\operatorname{span}(|g g\rangle,|e e\rangle)$ and $\mathcal{H}_{-}=\operatorname{span}(|g e\rangle,|e g\rangle)$ with corresponding parities $P_{+}=1$ and $P_{-}=-1$. Furthermore, such a measurement is not allowed to distinguish between states within the respective subspaces since this would destroy superpositions of states in either $\mathcal{H}_{+}$or $\mathcal{H}_{-}$. We consider a cQED system consisting of two superconducting charge qubits coupled to a single field mode of a superconducting stripline resonator [1,2]. Neglecting the influence of higher qubit states, the system is well described by the Tavis-Cummings Hamiltonian [26]:

$$
\begin{aligned}
H= & \omega_{r} a^{\dagger} a+\sum_{j=1}^{2} \frac{\omega_{q j}}{2} \sigma_{z}^{(j)}+\sum_{j=1}^{2} g_{j}\left[a^{\dagger} \sigma_{-}^{(j)}+a \sigma_{+}^{(j)}\right] \\
& +\left(a \epsilon_{m}^{*} e^{i \omega_{m} t}+a^{\dagger} \epsilon_{m} e^{-i \omega_{m} t}\right),
\end{aligned}
$$

where we have set $\hbar=1, \omega_{r}$ is the resonance frequency of the cavity mode, and $\epsilon_{m}$ is the drive amplitude of the measurement signal tuned to frequency $\omega_{m}$. The level splitting of qubit $j$ is given by $\omega_{q j}$, and $g_{j}$ is the coupling between the corresponding 
qubit and the cavity. To realize a qubit measurement, the system is operated in the dispersive regime, where the cavity and qubit frequencies are far detuned, $\lambda_{j}=g_{j} /\left|\Delta_{j}\right| \ll 1$, where $\Delta_{j}=\omega_{r}-\omega_{q j}$. In this limit, the system is well described by the second-order effective Hamiltonian [27]:

$$
\begin{aligned}
H_{\mathrm{eff}}= & \Delta_{r} a^{\dagger} a+\sum_{j=1}^{2} \chi_{j} \sigma_{z}^{(j)} a^{\dagger} a+\frac{\omega_{q j}+\chi_{j}}{2} \sigma_{z}^{(j)} \\
& +\left(a \epsilon_{m}^{*}+a^{\dagger} \epsilon_{m}\right)+J_{12}\left[\sigma_{-}^{(1)} \sigma_{+}^{(2)}+\sigma_{+}^{(1)} \sigma_{-}^{(2)}\right],
\end{aligned}
$$

written in the frame where the cavity degrees of freedom rotate at the frequency $\omega_{m}$ such that $\Delta_{r}=\omega_{r}-\omega_{m}$. The residual coupling between cavity and qubit $j$ is described by $\chi_{j}=$ $g_{j}^{2} / \Delta_{j}$, and $J_{12}=g_{1} g_{2}\left(\Delta_{1}+\Delta_{2}\right) / 2 \Delta_{1} \Delta_{2}$ is the qubit-qubit coupling mediated by virtual photons [27].

In the dispersive readout of $\mathrm{CQED}$, the joint state of the qubits is inferred from the homodyne signal coming from the transmitted microwaves through the cavity. Owing to the coupling between cavity and qubits, the phase and amplitude of the transmitted microwaves will depend on the state of the qubits $|i j\rangle, i, j=g, e$ with the field evolving into a coherent state $\left|\alpha_{i j}\right\rangle$ with amplitude $\alpha_{i j}$, which obeys the differential equation [28]

$$
\dot{\alpha}_{i j}(t)=-i \epsilon_{m}-i\left(\Delta_{r}+\chi_{i j}\right) \alpha_{i j}(t)-\frac{\kappa}{2} \alpha_{i j}(t) .
$$

Here $\chi_{i j}=\left\langle i j\left|\chi_{1} \sigma_{z}^{(1)}+\chi_{2} \sigma_{z}^{(2)}\right| i j\right\rangle$ is the coupling between the state $|i j\rangle$ and the cavity. In this case, the four different cavity states can be interpreted as pointer states $[29,30]$ of the measurement, where the state of the two qubits can be inferred from the state of the cavity field. To realize a parity measurement, we must make sure that the readout distinguishes between the two sets of field states $\left\{\alpha_{g g}, \alpha_{e e}\right\}$ and $\left\{\alpha_{g e}, \alpha_{e g}\right\}$ but not between the amplitudes within the sets. By choosing the couplings $g_{1}=g_{2}$ and detunings $\Delta_{1}=-\Delta_{2}$ and $\Delta_{r}=0$, the four different amplitudes in Eq. (3) satisfy

$$
\begin{gathered}
\alpha_{g g}(t)=\alpha_{e e}(t), \\
\operatorname{Re}\left[\alpha_{g e}(t)\right]=-\operatorname{Re}\left[\alpha_{e g}(t)\right], \\
\operatorname{Im}\left[\alpha_{g e}(t)\right]=\operatorname{Im}\left[\alpha_{e g}(t)\right],
\end{gathered}
$$

which can be seen in Fig. 1, where the quadrature $Q=$ $\operatorname{Im}\left[\alpha_{i j}(t)\right]$ is plotted against the in-phase component of the field $I=\operatorname{Re}\left[\alpha_{i j}(t)\right]$.

In a homodyne measurement, the field of the cavity is mixed with a local oscillator, with a relative phase $\phi$ defining the measured quadrature. By choosing $\phi=\pi / 2$, the $Q$ quadrature is measured as indicated in Fig. 1. In this case, the observer can distinguish between states of even and odd parity. The signal will, however, not contain information about the phase between states $\{|g e\rangle,|e g\rangle\}$ and $\{|g g\rangle,|e e\rangle\}$, as required by a parity measurement. Since the measurement eigenstates are not eigenstates of the qubit-qubit coupling [last term in Eq. (2)], we choose $\Delta_{1}=-\Delta_{2}$ to make this vanish.

\section{THE MODEL: EFFECTIVE TWO-QUBIT STOCHASTIC MASTER EQUATION}

The appropriate equation of motion to describe the evolution of the system conditioned on continuous homodyne

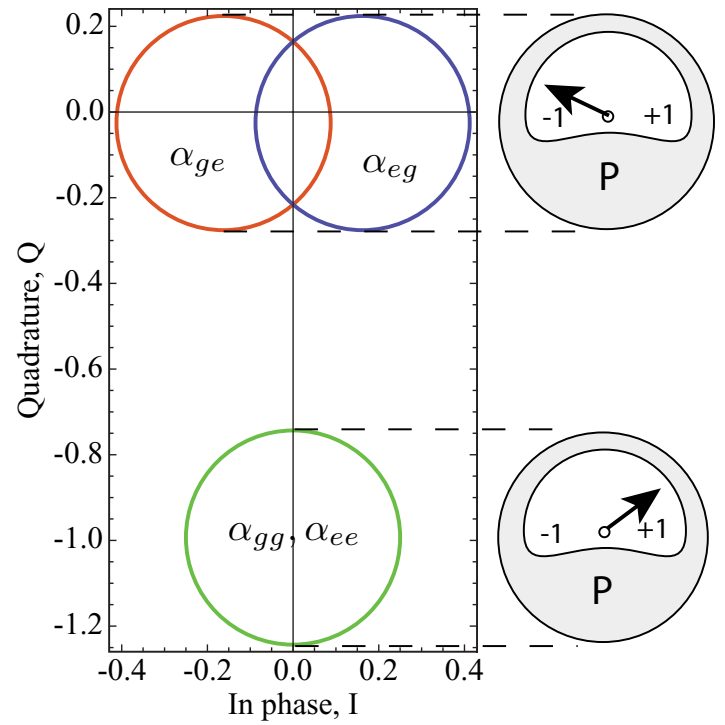

FIG. 1. (Color online) Phase space illustration of the stationary field states given in Eq. (3) with parameters $\epsilon_{m}=0.5 \kappa, \chi_{1}=-\chi_{2}=$ $1.5 \kappa$. By choosing the relative phase of the local oscillator to be $\phi=\pi / 2$, the homodyne readout measures the projection of the field on the $Q$ quadrature, allowing the observer to infer the parity of the qubits.

detection is given by the stochastic master equation [21]

$$
d \rho_{c}=\mathcal{L}_{\text {tot }} \rho_{c} d t+\sqrt{\kappa \eta} \mathcal{M}\left[a e^{-\phi}\right] \rho_{c} d W(t),
$$

where $\mathcal{L}_{\text {tot }} \rho_{c}$ is given by

$$
\begin{aligned}
\mathcal{L}_{\text {tot }} \rho_{c}= & -i\left[H_{\text {eff }}, \rho_{c}\right]+\sum_{j} \gamma_{1 j} \mathcal{D}\left[\sigma_{-}^{(j)}\right] \rho_{c}+\frac{\gamma_{\phi j}}{2} \mathcal{D}\left[\sigma_{z}^{(j)}\right] \rho_{c} \\
& +\kappa \mathcal{D}[a] \rho_{c}+\kappa \mathcal{D}\left[\sum_{j} \lambda_{j} \sigma_{-}^{(j)}\right] \rho_{c}
\end{aligned}
$$

where $\mathcal{D}[c] \rho=c \rho c^{\dagger}-1 / 2\left(c^{\dagger} c \rho+\rho c^{\dagger} c\right)$ is a dissipation super operator on Lindblad form [31], with the pure relaxation and dephasing rates of qubit $j$ given by $\gamma_{1 j}$ and $\gamma_{\phi j}$, respectively. The cavity damping rate is given by $\kappa$, and the last term of Eq. (6) describes the effect of Purcell damping [32]. The measurement back action on the system is described by the measurement superoperator

$$
\mathcal{M}[c] \rho_{c}=c \rho_{c}+\rho_{c} c^{\dagger}-\left\langle c+c^{\dagger}\right\rangle \rho_{c},
$$

where $\langle\cdot\rangle=\operatorname{tr}\left(\cdot \rho_{c}\right)$ and $d W(t)$ is defined as a Wiener increment completely characterized by its mean and variance [33]:

$$
\begin{aligned}
E[d W(t)] & =0, \\
E\left[d W(t)^{2}\right] & =d t .
\end{aligned}
$$

Here $E[\cdot]$ denotes the ensemble average taken over different realizations of the noise process $W(t)$. The homodyne current is given by

$$
j(t) d t=\sqrt{\kappa \eta}\left\langle a e^{-\phi}+a^{\dagger} e^{\phi}\right\rangle d t+d W(t),
$$

where $\eta$ is the efficiency at which the photons are detected.

As shown in Refs. [13] and [29] for multiple qubits and a single qubit, respectively, the cavity degrees of freedom can be traced out from Eq. (6) to obtain an effective master equation 
for the two qubits only. This can be done in the limit $\gamma_{1 j} \ll \kappa$, which is easily satisfied with current transmon qubits limited by the Purcell effect [34]. The elimination of cavity degrees of freedom is carried out by moving to a frame of reference given by the transformation

$$
\mathbf{P}=\sum_{i, j=g, e} \Pi_{i j} D\left[\alpha_{i j}\right],
$$

where the photon population in the cavity is essentially zero. Here $D[\alpha]=\exp \left(\alpha a^{\dagger}-\alpha^{*} a\right)$ is the displacement operator of the field [35] and $\Pi_{i j}=|i j\rangle\langle i j|$ are projection operators onto the respective basis states of the two qubit Hilbert space. This gives an effective master equation for the qubit degrees of freedom [13]:

$$
\begin{aligned}
d \rho_{c}= & -i\left[\sum_{j} \frac{\omega_{q j}+\chi_{j}}{2} \sigma_{z}^{(j)}, \rho_{c}\right] d t+\left(\sum_{j} \gamma_{1 j} \mathcal{D}\left[\sigma_{-}^{(j)}\right]\right. \\
& \left.+\frac{\gamma_{\phi j}}{2} \mathcal{D}\left[\sigma_{z}^{(j)}\right]+\kappa \mathcal{D}\left[\sum_{j} \lambda_{j} \sigma_{-}^{(j)}\right]\right) \rho d t \\
& +\sum_{i j, k l} \chi_{k l, i j}\left[\operatorname{Im}\left(\alpha_{i j}^{*} \alpha_{k l}\right)+i \operatorname{Re}\left(\alpha_{i j}^{*} \alpha_{k l}\right)\right] \Pi_{i j} \rho_{c} \Pi_{k l} d t \\
& +\sqrt{\kappa \eta} \mathcal{M}\left[\Pi_{\alpha} e^{-i \phi}\right] \rho_{c} d W(t),
\end{aligned}
$$

where $\chi_{i j, k l}=\chi_{i j}-\chi_{k l}$. The fifth term represents the dephasing induced by the measurement and the sixth term gives the ac Stark shift. The measurement operator $\Pi_{\alpha}$ is given by

$$
\begin{aligned}
\Pi_{\alpha} & =\sum_{i j} \alpha_{i j} \Pi_{i j} \\
& =\frac{1}{4}\left[\gamma_{z z} \sigma_{z}^{(1)} \sigma_{z}^{(2)}+\gamma_{z 1} \sigma_{z}^{(1)}+\gamma_{1 z} \sigma_{z}^{(2)}+\gamma_{11}\right],
\end{aligned}
$$

with the amplitudes

$$
\begin{gathered}
\gamma_{z z}=\alpha_{g g}-\alpha_{g e}-\alpha_{e g}+\alpha_{e e}, \\
\gamma_{z 1}=-\alpha_{g g}-\alpha_{g e}+\alpha_{e g}+\alpha_{e e}, \\
\gamma_{1 z}=-\alpha_{g g}+\alpha_{g e}-\alpha_{e g}+\alpha_{e e}, \\
\gamma_{11}=\alpha_{g g}+\alpha_{g e}+\alpha_{e g}+\alpha_{e e} .
\end{gathered}
$$

By choosing system parameters appropriately, the homodyne measurement along with single qubit rotations can thus be tuned to measure single as well as two-qubit operators, as discussed in Refs. [7-9,13]. The current can be expressed in terms of qubit operators as

$$
j(t) d t=\sqrt{\kappa \eta}\left\langle\Pi_{\alpha} e^{-i \phi}+\Pi_{\alpha}^{\dagger} e^{i \phi}\right\rangle d t+d W(t),
$$

which in the case of $\phi=\pi / 2$ reduces to

$$
j_{\mathrm{ij}}(t) d t=2 \sqrt{\kappa \eta} \operatorname{Im}\left(\alpha_{i j}\right) d t+d W(t)
$$

for the basis states $|i j\rangle$. With our choice of parameters, $\operatorname{Im}\left(\alpha_{g g}\right)=\operatorname{Im}\left(\alpha_{e e}\right)$ and $\operatorname{Im}\left(\alpha_{g e}\right)=\operatorname{Im}\left(\alpha_{e g}\right)$ such that the measurement does not distinguish between states within $\mathcal{H}_{+}$and $\mathcal{H}_{-}$, as required for a parity measurement.

\section{MEASUREMENT-INDUCED DEPHASING}

With the field amplitudes given in Eq. (4) and the LO phase given by $\phi=\pi / 2$, the measurement operator can be rewritten as

$$
\begin{aligned}
\mathcal{M}\left[-i \Pi_{\alpha}\right] \rho_{c}= & \frac{\beta}{2} \mathcal{M}\left[\Pi_{+}-\Pi_{-}\right] \rho_{c} \\
& -i \operatorname{Re}\left(\alpha_{g e}\right)\left[\Pi_{g e}-\Pi_{e g}, \rho_{c}\right],
\end{aligned}
$$

where

$$
\begin{aligned}
& \Pi_{+}=|g g\rangle\langle g g|+| e e\rangle\langle e e|, \\
& \Pi_{-}=|g e\rangle\langle g e|+| e g\rangle\langle e g|
\end{aligned}
$$

are projection operators onto $\mathcal{H}_{+}$and $\mathcal{H}_{-}$and $\beta$ is the difference field:

$$
\beta=\operatorname{Im}\left(\alpha_{g g}\right)-\operatorname{Im}\left(\alpha_{g e}\right) .
$$

The first term of Eq. (16) is associated with information gain and simply expresses the fact that the parity measurement tends to localize an initial state in one of the subspaces $\mathcal{H}_{+}$or $\mathcal{H}_{-}$at a rate given by

$$
\Gamma_{\mathrm{m}}(t)=\kappa \eta \beta(t)^{2},
$$

which can be taken as a definition of the measurement rate. The second term represents a stochastic phase between the states $|g e\rangle$ and $|e g\rangle$ accumulated during the measurement. This, together with the dephasing term $\propto \operatorname{Im}\left(\alpha_{i j}^{*} \alpha_{k l}\right)$ given in Eq. (11), represents the unwanted back action of the measurement, which we now analyze. Because of the nonlinear nature of conditional state evolution, it is not possible to analytically solve Eq. (11) in the general case. However, in the case when the state has been projected onto $\mathcal{H}_{ \pm}$, the nonlinear part vanishes, and Eq. (11) reduces to

$$
d \rho_{c}(t)=\frac{\Gamma_{g e, e g}}{2} \mathcal{D}\left[\Sigma_{z}\right] \rho_{c}(t) d t-i \sqrt{\kappa \eta \Omega} \mathcal{K} \rho_{c}(t) d W(t),
$$

where $\quad \Sigma_{z}=\Pi_{g e}-\Pi_{e g}, \quad \mathcal{K} \rho_{c}=\left[\Sigma_{z}, \rho\right], \quad \Gamma_{g e, e g}=$ $4 \chi \operatorname{Im}\left(\alpha_{g e}^{*} \alpha_{e g}\right)$, and $\Omega=\operatorname{Re}^{2}\left(\alpha_{g e}\right)$. Since the focus of this analysis is on the back action of the measurement, we have neglected the effect of pure relaxation and dephasing as well as Purcell damping. In the numerical simulations presented in Sec. VI, however, these effects are taken into account. Furthermore, we disregard the deterministic rotation of the phase $\propto \operatorname{Re}\left(\alpha_{i j}^{*} \alpha_{k l}\right)$ given in Eq. (11) since this can be undone regardless of the measurement outcome. Equation (20) can be solved analytically [33], yielding

$$
\begin{aligned}
\rho_{c}(t)= & \exp \left[\frac{1}{2} \int_{0}^{t} \Gamma_{g e, e g}(s) \mathcal{D}\left[\Sigma_{z}\right]+\kappa \eta \Omega(s) \mathcal{K}^{2} d s\right. \\
& \left.-i \int_{0}^{t} \sqrt{\kappa \eta \Omega(s)} d W(s) \mathcal{K}\right] \rho_{c}(0) \\
= & \exp \left\{\frac{1}{2} \int_{0}^{t}\left[\Gamma_{g e, e g}(s)-2 \kappa \eta \Omega(s)\right] \mathcal{D}\left[\Sigma_{z}\right] d s\right. \\
& \left.-i \int_{0}^{t} \sqrt{\kappa \eta \Omega(s)} d W(s) \mathcal{K}\right\} \rho_{c}(0),
\end{aligned}
$$

where we have used the fact that $\mathcal{K}^{2} \rho=-2 \mathcal{D}\left[\Sigma_{z}\right] \rho$. Given this, we see two effects of the measurement. First, the third line 
in Eq. (21) gives the dephasing rate $\Gamma_{c}$ for a single trajectory:

$$
\Gamma_{c}(t)=\Gamma_{g e, e g}(t)-2 \kappa \eta \Omega(t) .
$$

The second effect is given by the last term in Eq. (21), which gives rise to a stochastic phase between $|g e\rangle$ and $|e g\rangle$. Since this phase varies between different measurements, the ensemble-averaged state is mixed with the corresponding dephasing rate given by $\Gamma_{g e, e g}$. As a measure of the readout fidelity, we take the ratio between the measurement and dephasing rate as $t \rightarrow \infty$ :

$$
\frac{\Gamma_{g e, e g}}{\Gamma_{\mathrm{m} \mid t \rightarrow \infty}}=\frac{\kappa^{2}}{8 \eta \chi^{2}},
$$

which shows that the dispersive readout only works as a perfect parity measurement in the large coupling limit $\chi \gg \kappa$. This limit may, however, be experimentally hard to reach [13], and it is therefore desirable to seek alternative methods to improve on this ratio. Considering the same ratio, but for a single trajectory, gives the result

$$
{\frac{\Gamma}{\Gamma_{\mathrm{m} \mid t \rightarrow \infty}}}=\frac{(1-\eta) \kappa^{2}}{8 \eta \chi^{2}},
$$

which vanishes in the case of a perfect measurement $\eta=1$. This can be understood by looking at the two terms in Eq. (22). In Fig. 2, we see that for a perfect measurement, $\Gamma_{c} \rightarrow 0$ as $t \rightarrow \infty$, which is because the measurement current contains information about the photon fluctuations. As $t \rightarrow \infty$, the observer can track the photon fluctuations by looking at the homodyne current such that no information is lost into the environment. Given this information, the observer can in principle undo the stochastic phase with a resulting pure ensemble-averaged state. From Fig. 2, we also see that for the dispersive measurement, there is initial dephasing in the system not caught when taking the limit $t \rightarrow \infty$. This is given by

$$
\lim _{\tau \rightarrow \infty} \int_{0}^{\tau} \Gamma_{c}(s) d s=\frac{128 \epsilon^{2} \chi^{2}}{\left(\kappa^{2}+16 \chi^{2}\right)^{2}}, \quad \eta=1,
$$

which simply reflects the fact that the homodyne measurement is not able to record information about the initial photon

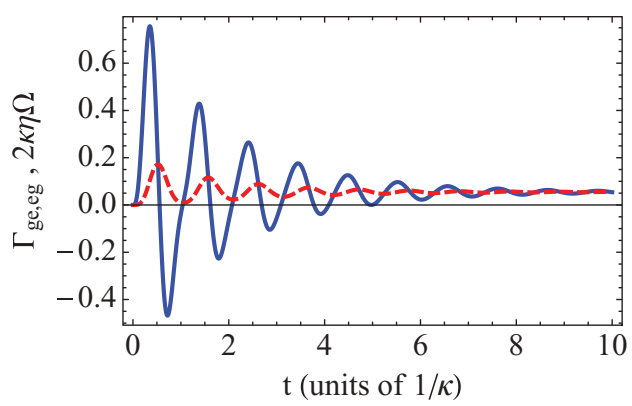

FIG. 2. (Color online) The dephasing rates $\Gamma_{g e, e g}$ (solid blue line) and $2 \kappa \eta \Omega$ (dashed red line). Initially, the system is prone to dephasing with $\Gamma_{g e, e g}>2 \kappa \eta \Omega$. This reflects the fact that initial fluctuations in the photon number cannot be captured by the homodyne measurement. As $t \rightarrow \infty, \Gamma_{g e, e g} \rightarrow 2 \kappa \eta \Omega$, with all the unwanted back action given by a stochastic phase between the basis states $|g e\rangle$ and $|e g\rangle$. The parameters are $\epsilon_{m}=\kappa, \chi_{1}=-\chi_{2}=3 \kappa$, and $\eta=1$. fluctuations because of the finite response time of the cavity. Because of this, inevitable ${ }^{1}$ dephasing occurs with the final state being mixed even for a single trajectory. The dephasing in Eq. (25) is a decreasing function of $\chi$ since the number of photons in the cavity interacting with the qubits decreases as the coupling to the qubits shifts the cavity frequency. Moreover, the dephasing decreases with increasing $\kappa$. This is simply because a faster response time of the cavity allows for a more efficient measurement of the initial photon fluctuations. For an imperfect measurement with $\eta<1$, the dephasing rate of a single trajectory increases linearly with $\eta$ such that $\Gamma_{c} \rightarrow \Gamma_{g e, e g}$ as $\eta \rightarrow 0$. Current signal to noise ratios of dispersive qubit readout in $\mathrm{cQED}$ correspond roughly to $\eta \sim 1 /(5-25)$, a figure which will, it is hoped, increase in the near future [23-25].

From Eq. (24), we note that the measurement fidelity of a single trajectory is far better than that given for the ensembleaveraged state in Eq. (23). This is because the stochastic phase does not contribute to the dephasing for a single trajectory. From this it is clear that the purity of the ensemble-averaged state can be drastically increased if the observer is able to undo the stochastic phase after each measurement shot. In this case, the dispersive readout approaches a true parity measurement even in the case of moderate coupling between qubits and cavity. In the next section, we present such a scheme based on state estimation and feedback. We show that this can be done without knowledge of the initial state.

\section{INCREASING MEASUREMENT FIDELITY BY STATE ESTIMATION}

Information about the stochastic phase discussed in Sec. IV cannot be extracted from the measurement current directly. We may, however, use the recorded current to extract the noise process $W(t)$ for a given trajectory. This can be used as an input to Eq. (11) to calculate an estimate $\tilde{\rho}_{c}(t)$ of the state $[18,19]$. Unfortunately, this procedure requires knowledge of the initial state $\rho_{c}(0)$, which is typically unknown in a measurement situation. We now show that no information about the initial state is needed if we are only interested in the accumulated stochastic phase.

Given an unknown initial state, the observer will make a wrong estimate $d \tilde{W}(t)$ of the true noise process:

$$
\begin{aligned}
d \tilde{W}(t)= & d W(t)+\sqrt{\kappa \eta} \operatorname{tr}\left\{\left(\Pi_{\alpha} e^{-i \phi}+\Pi_{\alpha}^{\dagger} e^{i \phi}\right)\right. \\
& \left.\times\left[\rho_{c}(t)-\tilde{\rho}_{c}(t)\right]\right\} d t,
\end{aligned}
$$

where $\rho_{c}(t)$ is the true state of the system and $\tilde{\rho}_{c}(t)$ is the guessed estimate. We write the true state as a mix of odd- and even-parity states:

$$
\rho_{c}(t)=p(t) \rho_{+}(t)+[1-p(t)] \rho_{-}(t),
$$

where $\rho_{+}(t) \in \mathcal{H}_{+}$and $\rho_{-}(t) \in \mathcal{H}_{-}$. The probability $p(t) \in$ $\{0,1\}$ describes the conditional evolution toward one of the respective subspaces. We choose the estimated state to initially reside in the $\mathcal{H}_{-}$subspace such that the subsequent state can

\footnotetext{
${ }^{1}$ At least for a sudden onset of the measurement signal, which we consider here.
} 
be written

$$
\tilde{\rho}_{c}(t)=\tilde{\rho}_{-}(t) .
$$

Considering the measurement part of Eq. (11) only, we can write three coupled equations for the $|g e\rangle\langle e g|$ matrix element of $\rho_{c}, \tilde{\rho}_{c}$, and $p(t)$ :

$$
\begin{gathered}
d \rho_{g e, e g}=-2 \sqrt{\kappa \eta}\left[\beta p+i \operatorname{Re}\left(\alpha_{g e}\right)\right] \rho_{g e, e g} d W(t), \\
d \tilde{\rho}_{g e, e g}=-i 2 \sqrt{\kappa \eta}[2 \sqrt{\kappa \eta} \beta p d t+d W(t)] \operatorname{Re}\left(\alpha_{g e}\right) \tilde{\rho}_{g e, e g}, \\
d p(t)=-2 \sqrt{\kappa \eta} \beta p(1-p) d W(t) .
\end{gathered}
$$

Given the solution for $p(t)$, we can calculate the phases $\phi=$ $\arg \left(\rho_{g e, e g}\right)$ and $\tilde{\phi}=\arg \left(\tilde{\rho}_{g e, e g}\right)$ of the true and estimated state, respectively:

$$
\begin{aligned}
\phi(t)= & \tilde{\phi}(t)=-4 \kappa \eta \int_{0}^{t} \beta(s) \operatorname{Re}\left[\alpha_{g e}(s)\right] p(s) d t \\
& -2 \sqrt{\kappa \eta} \int_{0}^{t} \operatorname{Re}\left[\alpha_{g e}(s)\right] d W(t) .
\end{aligned}
$$

Hence we see that the accumulated phase of the estimated state equals that of the true state! This is the main result of this article. This is consistent with the result of Ref. [20], where a strong projective homodyne measurement is considered. This can be seen as the limiting case when $\kappa \rightarrow \infty$ of our analysis. Since the conditional evolution does not depend on whether the true state is a mix or superposition between even- and odd-parity states, the main result in Eq. (30) holds equally well when the true state is an arbitrary superposition state. Hence the method of undoing the accumulated phase can be applied not only in the context of, for example, entanglement generation, as considered in Ref. [13], but also in a real measurement situation where the initial state is unknown. This will considerably reduce the unwanted dephasing caused by the measurement back action.

\section{RESULTS}

To quantify how much the method of state estimation and subsequent subtraction of the accumulated phase increases the purity of the postmeasurement state, we perform numerical

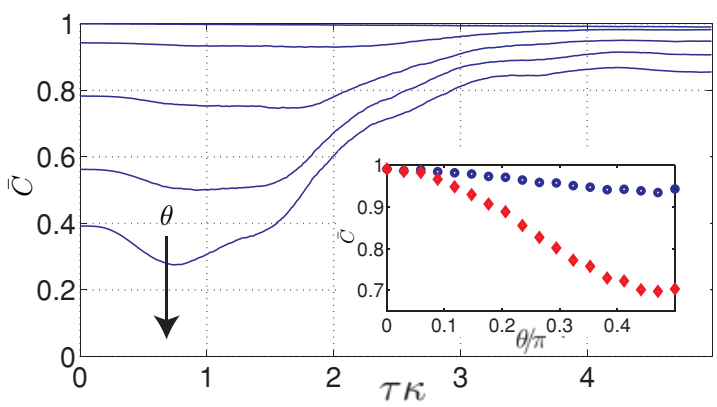

FIG. 3. (Color online) Average concurrence $\bar{C}$ as a function of time plotted for five initial states on the form given in Eq. (33). The angle $\theta$ is in the range $\theta \in\{0, \pi / 4\}$ (increasing from top to bottom). The inset shows $\bar{C}$ at the final time of the measurement, with (blue circles) and without (red diamonds) the phase subtracted. The parameters are given by $\epsilon_{m}=\kappa, \chi_{1}=-\chi_{2}=3 \kappa, g_{1}=-g_{2}=100 \kappa$, $\gamma_{1 j}=\gamma_{\phi j}=0$, and $\eta=1$.

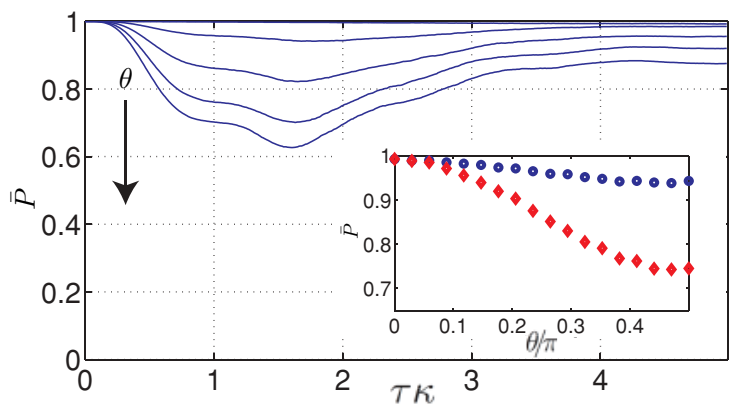

FIG. 4. (Color online) Average purity $\bar{P}$ as a function of time plotted for the same initial states as in Fig. 3. The inset shows $\bar{P}$ at the final time of the measurement, with (blue circles) and without (red diamonds) the phase subtracted. The parameters are the same as in Fig. 3.

simulations of Eq. (11). This is necessary since the full dynamics of Eq. (11) cannot be solved analytically. To distinguish the odd- and even-parity states, we use the integrated current

$$
s(t)=\int_{0}^{t} j(s) d s
$$

and assign the postmeasurement states to $\mathcal{H}_{+}$or $\mathcal{H}_{-}$if $s(t)$ satisfies $s(t)>s_{\text {th }}$ or $s(t)<s_{\text {th }}$, where $s_{\text {th }}$ is a threshold given by the average current $s_{\mathrm{th}}=\sqrt{\kappa \eta} \int_{0}^{t}\left[\operatorname{Im}\left(\alpha_{g g}\right)+\operatorname{Im}\left(\alpha_{g e}\right)\right] d s$. To quantify the fidelity of the parity measurement and the estimation scheme, we define the average concurrence [36] $\bar{C}=C\left(E\left[\rho_{c}\right]\right)$, purity [14] $\bar{P}=\operatorname{tr}\left[\left(E\left[\rho_{c}\right]\right)^{2}\right]$, and fidelity [14, 37]

$$
\bar{F}=\frac{n_{+}\left\langle\psi_{+}\left|E_{+}\left[\rho_{c}\right]\right| \psi_{+}\right\rangle+n_{-}\left\langle\psi_{-}\left|E_{-}\left[\rho_{c}\right]\right| \psi_{-}\right\rangle}{n_{+}+n_{-}},
$$

where $n_{+} / n_{-}$is the number of trajectories assigned to measurement outcome \pm and $E_{ \pm}\left[\rho_{c}\right]$ is the ensemble average over the respective states. The states $\left|\psi_{ \pm}\right\rangle$are even- and odd-parity states with the initial state given by

$$
|\psi\rangle=\cos (\theta)\left|\psi_{+}\right\rangle+\sin (\theta)\left|\psi_{-}\right\rangle .
$$

The initial estimated state is correspondingly given by $|\tilde{\psi}\rangle=$ $\left|\tilde{\psi}_{-}\right\rangle$, where $\left|\tilde{\psi}_{-}\right\rangle$need not equal $\left|\psi_{-}\right\rangle .^{2}$ We plot $\bar{C}, \bar{P}$, and $\bar{F}$ in Figs. 3, 4, and 5 for different values of $\theta$. In each figure, the inset shows the value of the respective quantity at the final time with and without the subtraction of the accumulated phase. It is clear that the state estimation allows us to subtract the stochastic phase in each measurement run, resulting in a drastic improvement of the state fidelity across all three measures.

The effect of limited measurement efficiency $\eta<1$ can be seen in Fig. 6 , where the average concurrence $\bar{C}$ at time $\tau_{m}=5 / \kappa$ is plotted for $\eta \in[0,1]$. Clearly the method of state estimation never decreases the fidelity of the postmeasurement state beyond that of the state obtained without state estimation. It is, however, clear that one needs to exploit the recent

\footnotetext{
${ }^{2}$ In the simulations, the initial states were chosen as $\left|\psi_{+}\right\rangle=1 / \sqrt{2}(|g g\rangle+|e e\rangle), \quad\left|\psi_{-}\right\rangle=1 / \sqrt{2}(|g e\rangle+\exp (i \pi / 4)|e g\rangle)$, and $\left|\tilde{\psi}_{-}\right\rangle=1 / \sqrt{2}(|g e\rangle+|e g\rangle)$.
} 




FIG. 5. (Color online) Average fidelity $\bar{F}$ as a function of time plotted for the same initial states as in Fig. 3. The inset shows $\bar{F}$ at the final time of the measurement, with (blue circles) and without (red diamonds) the phase subtracted. The parameters are the same as in Fig. 3.

advances in low-noise microwave amplifiers for this method to provide a significant increase in fidelity [23-25].

\section{A. Imperfect measurement record}

We note that our estimation scheme relies on the assumption that we can make a perfect record of the homodyne current, which is impossible in the case of a detector with limited bandwidth. To give an estimate of the effect of such measurement imperfections, we therefore consider the situation in which the estimated state is based on the value of the time-averaged current:

$$
j_{\mathrm{lp}}(t)=\frac{1}{\tau} \int_{t-\tau}^{t} j(s) d s,
$$

where $\tau$ is the time window over which $j(t)$ is averaged. In this case, the value of the estimated phase will differ from the true value, and the averaging over measurement results will reduce the purity of the state. In Fig. 7, we plot the imaginary and real part of $\rho_{g e, e g}$ of the postmeasurement density matrix for each trajectory. The initial state is given by $|\psi\rangle=1 / \sqrt{2}(|g e\rangle+$ $\exp (i \pi / 4)|e g\rangle)$.

We see that the state estimation scheme successfully recovers the true phase of the initial state even in the case where we do not have access to a perfect measurement record. As $\tau$ increases, the fidelity goes down, as expected, but does not reach the value obtained without state estimation even

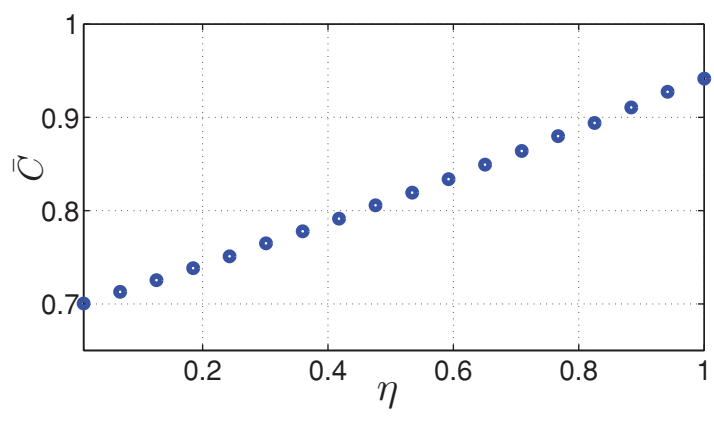

FIG. 6. (Color online) Average concurrence $\bar{C}$ as a function of measurement efficiency $\eta$. The initial state is taken to be $\left|\psi_{-}\right\rangle=1 / \sqrt{2}(|g e\rangle+\exp (i \pi / 4)|e g\rangle)$, and the measurement time is $\tau_{m}=5 / \kappa$. The remaining parameters are the same as in Fig. 3 .
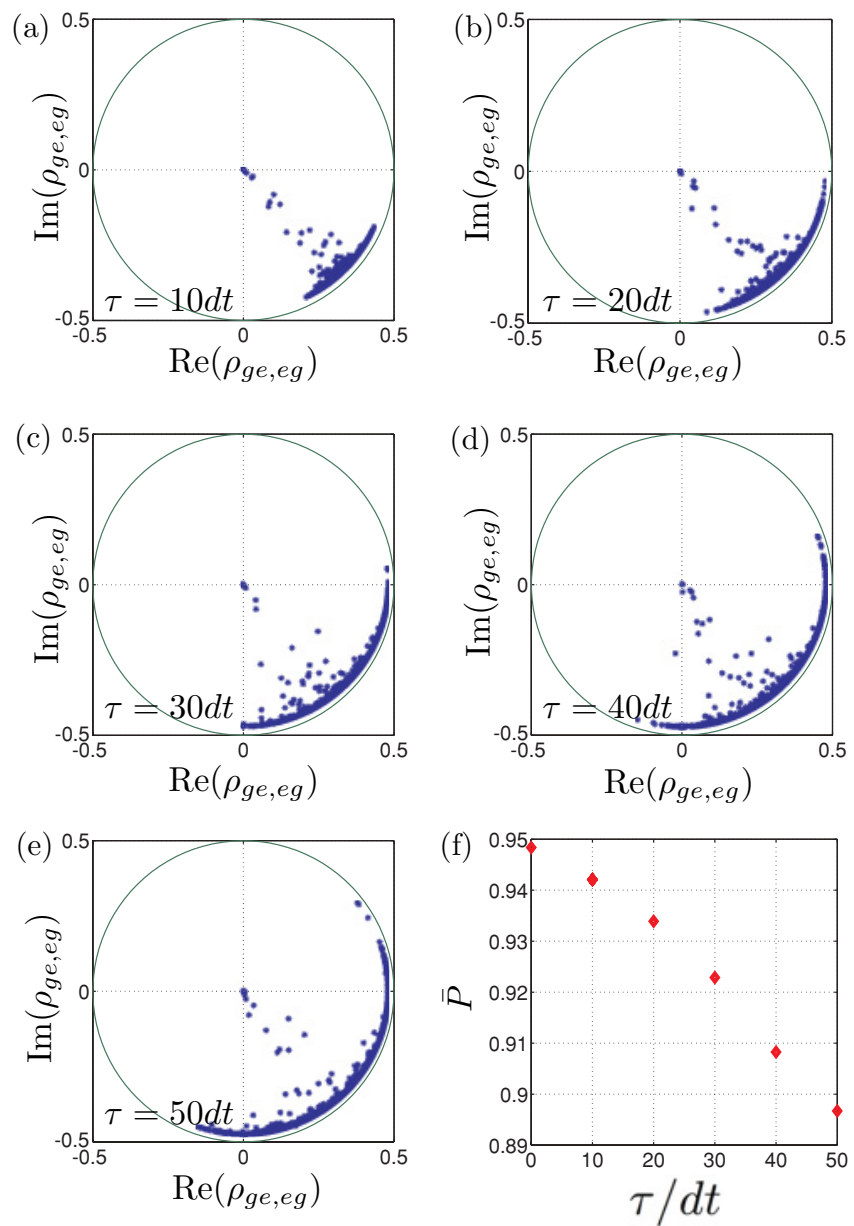

FIG. 7. (Color online) (a-e) $\operatorname{Im}\left(\rho_{g e, e g}\right)$ of the postmeasurement states against $\operatorname{Re}\left(\rho_{g e, e g}\right)$. The initial state is given by $|\psi\rangle=$ $1 / \sqrt{2}(|g e\rangle+\exp (i \pi / 4)|e g\rangle)$. As $\tau$ increases, the estimation of the accumulated phase becomes worse, and the phase of the state is distributed around $\phi=-\pi / 4$. (f) Average purity plotted as function of $\tau$. Even for $\tau=50 d t$, the purity does not reach the corresponding value obtained in the case without state estimation and feedback. The parameters are the same as in Fig. 3.

for $\tau=50 d t$. We can understand this robustness from the observation that the values of $\rho_{g e, e g}$ are distributed on a circle arc which makes the decrease of purity quadratic in the phase uncertainty. This can also be seen in the initial slope in Fig. 7.

\section{CONCLUSION}

In conclusion, we have performed an analysis of a two-qubit parity measurement based on dispersive readout in cQED. In particular, we analyzed the back action on the two qubits and found two qualitatively different contributions. One is an unavoidable dephasing in one of the parity subspaces, arising during the transient time of switching on the measurement. This dephasing occurs in the process of entangling the state of the driven cavity with the two qubits. The other part is a stochastic rotation of the phase in the same subspace, which persists during the whole measurement. We discussed how the latter can be determined from the full 
measurement time trace using the method of state estimation. Quite surprisingly, we found that the outcome of this phase determination process is independent of the initial state in the state estimation procedure, making it useful in the situation of a parity measurement, where the initial state, by definition, is unknown. Finally, we show that the feedback process is rather robust toward imperfections in the measurement record. With improved measurement efficiencies, this analysis opens for the realization of high-fidelity parity measurements in cQED using moderate values of the coupling strength between the qubits and the cavity.

\section{ACKNOWLEDGMENTS}

We thank K. Lalumière, T. Stace, G. Milburn, and C. Wilson for valuable discussions. This work is supported by the European Commission through the IST-015708 EuroSQIP integrated project and by the Swedish Research Council.
[1] A. Blais, R. S. Huang, A. Wallraff, S. M. Girvin, and R. J. Schoelkopf, Phys. Rev. A 69, 062320 (2004).

[2] A. Wallraff, D. I. Shuster, A. Blais, L. Frunzio, R.-S. Huang, J. Majer, S. Kumar, S. M. Girvin, and R. J. Shoelkopf, Nature (London) 431, 162 (2004).

[3] G. Wendin and V. S. Shumeiko, in Handbook of Theoretical and Computational Nanotechnology, edited by M. Rieth and W. Schommers (American Scientific, Los Angeles, 2006).

[4] J. Q. You and F. Nori, Phys. Today 58, 42 (2005).

[5] J. Clarke and F. K. Wilhelm, Nature (London) 453, 1031 (2008).

[6] J. M. Chow, J. M. Gambetta, L. Tornberg, J. Koch, L. S. Bishop, A. A. Houck, B. R. Johnson, L. Frunzio, S. M. Girvin, and R. J. Schoelkopf, Phys. Rev. Lett. 102, 090502 (2009).

[7] L. DiCarlo et al., Nature (London) 460, 240 (2009).

[8] S. Filipp et al., Phys. Rev. Lett. 102, 200402 (2009).

[9] J. M. Chow, L. DiCarlo, J. M. Gambetta, A. Nunnenkamp, L. S. Bishop, L. Frunzio, M. H. Devoret, S. M. Girvin, and R. J. Schoelkopf, Phys. Rev. A 81, 062325 (2010).

[10] D. P. DiVincenzo, Fortschr. Phys. 48, 771 (2000).

[11] C. W. J. Beenakker, D. P. DiVincenzo, C. Emary, and M. Kindermann, Phys. Rev. Lett. 93, 020501 (2004).

[12] H.-A. Engel and D. Loss, Science 309, 586 (2005).

[13] K. Lalumière, J. M. Gambetta, and A. Blais, Phys. Rev. A 81, 040301(R) (2010).

[14] M. A. Nielsen and I. L. Chuang, Quantum Computation and Information (Cambridge University Press, Cambridge, 2000).

[15] C. Ahn, A. C. Doherty, and A. J. Landahl, Phys. Rev. A 65, 042301 (2002).

[16] M. Sarovar, C. Ahn, K. Jacobs, and G. J. Milburn, Phys. Rev. A 69, 052324 (2004).

[17] J. von Neumann, Mathematical Foundations of Quantum Mechanics (Princeton University Press, Princeton, 1955).

[18] A. C. Doherty, S. Habib, K. Jacobs, H. Mabuchi, and S. M. Tan, Phys. Rev. A 62, 012105 (2000).
[19] A. C. Doherty and K. Jacobs, Phys. Rev. A 60, 2700 (1999).

[20] S. D. Barrett, P. Kok, K. Nemoto, R. G. Beausoleil, W. J. Munro, and T. P. Spiller, Phys. Rev. A 71, 060302(R) (2005).

[21] H. M. Wiseman and G. J. Milburn, Phys. Rev. A 47, 642 (1993).

[22] B. Yurke, Phys. Rev. A 29, 408 (1984).

[23] M. A. Castellanos-Beltran, K. D. Irwin, G. C. Hilton, L. R. Vale, and K. W. Lehnert, Nat. Phys. 4, 929 (2008).

[24] M. Mück, C. Welzel, and J. Clarke, Appl. Phys. Lett. 82, 3266 (2003).

[25] N. Bergeal, F. Schackert, M. Metcalfe, R. Vijay, V. E. Manucharyan, L. Frunzio, D. E. Prober, R. J. Schoelkopf, S. M. Girvin, and M. H. Devoret, Nature (London) 465, 64 (2010).

[26] M. Tavis and F. W. Cummings, Phys. Rev. 170, 379 (1968).

[27] A. Blais, J. Gambetta, A. Wallraff, D. I. Schuster, S. M. Girvin, M. H. Devoret, and R. J. Schoelkopf, Phys. Rev. A 75, 032329 (2007).

[28] J. Gambetta, A. Blais, D. I. Schuster, A. Wallraff, L. Frunzio, J. Majer, M. H. Devoret, S. M. Girvin, and R. J. Schoelkopf, Phys. Rev. A 74, 042318 (2006).

[29] J. Gambetta, A. Blais, M. Boissonneault, A. A. Houck, D. I. Schuster, and S. M. Girvin, Phys. Rev. A 77, 012112 (2008).

[30] W. H. Zurek, Rev. Mod. Phys. 75, 715 (2003).

[31] G. Lindblad, Commun. Math. Phys. 48, 119 (1976).

[32] E. M. Purcell, H. C. Torrey, and R. V. Pound, Phys. Rev. 69, 37 (1946)

[33] F. C. Klebaner, Introduction to Stochastic Calculus with Applications (Imperial College Press, London, 2005).

[34] A. A. Houck et al., Phys. Rev. Lett. 101, 080502 (2008).

[35] C. C. Gerry and P. L. Knight, Introductory Quantum Optics (Cambridge University Press, Cambridge, 2005).

[36] W. K. Wootters, Phys. Rev. Lett. 80, 2245 (1998).

[37] S. M. Barnett, Quantum Information (Oxford University Press, Oxford, 2009). 